\title{
Star Formation in Southern Seyfert Galaxies
}

\author{
Duncan A. Forbes \\ School of Physics and Astronomy, University of Birmingham, Birmingham, B15 2TT \\ Email: forbes@star.sr.bham.ac.uk \\ R. P. Norris \\ Australia Telescope National Facility, CSIRO Radiophysics Laboratory, P.O. Box 76, Epping, NSW 2121, \\ Australia \\ Email: rnorris@atnf.csiro.au
}

\begin{abstract}
We have produced radio maps, using the ATCA, of the central regions of six southern Seyfert 2 galaxies (NGC 1365, 4945, 6221, 6810, 7582, and Circinus) with circumnuclear star formation, to estimate the relative contribution of star formation activity compared to activity from the active galactic nucleus (AGN). The radio morphologies range from extended diffuse structures to compact nuclear emission, with no evidence, even in the relatively compact sources, for synchrotron self-absorption. In each case the radio to far-infrared (FIR) ratio has a value consistent with star formation, and in all but one case the radio to [FeII] ratio is also consistent with star formation. We derive supernova rates and conclude that, despite the presence of a Seyfert nucleus in these galaxies, the radio, FIR, and [FeII] line emission are dominated by processes associated with the circumnuclear star formation (i.e. supernova remnants and H II regions) rather than with the AGN.
\end{abstract}

Subject headings: galaxies: individual - galaxies: nuclei - radio continuum: galaxies

\section{Introduction}

Circumnuclear star formation is common in Seyfert galaxies, but the relationship between the Seyfert nucleus and the surrounding star formation is not well understood (see Filippenko 1992), and both evolutionary and causal relationships have been suggested. For example, a nuclear starburst may develop into a massive star cluster or black hole (Norman \& Scoville 1988), or conversely the energy output from an active galactic nucleus (AGN) may trigger circumnuclear star formation (Sanders \& Bania 1976). More recently, studies have indicated that star formation is occurring in and around the torus (e.g. Cid Fernandes \& Terlevich 1992; Davies et al. 1997) that is thought to dictate the type of Seyfert nucleus we observe.

Dust is a common feature of the circumnuclear environment of active galaxies. At optical wavelengths, it can obscure our view of the nucleus and hide any evidence of an AGN. However, radio observations are not only unaffected by dust but also have the advantage of high spatial resolution, and can be important in understanding the role of the various processes in active galaxies. For example, starburst galaxies generally have diffuse radio morphologies dominated by synchrotron emission from cosmic rays accelerated by supernovae, while Seyfert galaxies sometimes exhibit well-collimated radio jets and a compact nuclear radio source (see review by Condon 1992). 
High-resolution radio synthesis images, with sub-arcsec resolution, have found compact sources at the nucleus, and extended emission consisting of radio jets, ouflows, diffuse emission, and discrete sources such as HII regions and SNRs (e.g. Ulvestad \& Wilson 1984; Kronberg et al. 1985; Antonucci \& Ulvestad 1988; Carral, Turner \& Ho 1990; Condon et al. 1991; Forbes et al. 1994; Collison et al. 1994; Sandqvist et al. 1995).

High spatial resolution, multi-frequency radio observations can also be used to test the idea of advection-dominated accretion flows around black holes. For such flows the radio emission depends strongly on the mass of the black hole and is characterised by an inverted radio spectrum (Yi \& Boughn 1997).

Here we present 3 and $6 \mathrm{~cm}$ radio continuum images from the Australia Telescope Compact Array (ATCA) of 6 such galaxies which show evidence for narrow high excitation optical forbidden lines, and are classified as Seyfert 2s. In the case of NGC 1365, broad lines have also been detected from the nuclear region. All six galaxies show evidence for circumnuclear star formation, and several are barred. We discuss the radio morphologies of these galaxies and possible emission mechanisms, and we compare the radio data with that from other wavelengths to assess the relative contributions to the radio flux from star formation and the AGN.

\section{Observations and Data Reduction}

The observations were carried out using the Australia Telescope Compact Array at $4.79 \mathrm{GHz}(6$ $\mathrm{cm})$ and $8.64 \mathrm{GHz}(3 \mathrm{~cm})$ simultaneously. The observational parameters are given in Table 1 . The same amplitude calibrator (1934-638) was observed with each galaxy, with an assumed flux density of $5.83 \mathrm{Jy}$ at $6 \mathrm{~cm}$ and $2.84 \mathrm{Jy}$ at $3 \mathrm{~cm}$. Observations of the target galaxy and a phase calibrator were alternated throughout the observing run, with a total of about 10 hours spent on-source on each galaxy. The data were edited, calibrated and CLEANed using the AIPS software package. The typical half-power beam-width (HPBW) of the final images are $2 \operatorname{arcsec}$ at $6 \mathrm{~cm}$ and 1 arcsec at $3 \mathrm{~cm}$.

Because these observations and their analysis were optimised for studying the nuclear region, they are relatively insensitive to extended emission. Thus our images are primarily of the nuclear region, and we will therefore not show any smooth extended structure on scales of tens of arcsec.

\section{Results and Discussion}

In Figures 1 to 12 we show the 3 and $6 \mathrm{~cm}$ radio images for the six galaxies in our sample. These galaxies do not represent a complete sample in any sense, but rather were chosen as relatively well-known southern Seyfert 2 galaxies that generally lacked high resolution radio maps. Our radio data for the Circinus galaxy have been presented elsewhere along with near-infrared line imaging (Davies et al. 1997), although we include it here for comparison purposes with the other galaxies. The images show a variety of radio morphologies which range from Circinus, with a strong, compact nucleus, to NGC 1365, with a extended region of discrete sources or hot-spots. The beam shape is shown in the lower left of each image.

Care is needed when interpreting these images, as (a) our observations are optimised for studying the nuclear region, and so our images will not correctly represent the extended diffuse emission from the disk of 
the galaxy, and (b) most of the galaxies have high inclinations so that confusion effects may play some role in the observed radio morphology.

Flux measurements in 2 arcsec and 6 arcsec diameter apertures for each galaxy are given in Table 2. We also list the $6 \mathrm{~cm}$ to $3 \mathrm{~cm}$ spectral index after smoothing the $3 \mathrm{~cm}$ image to match the $6 \mathrm{~cm}$ resolution. The spectral indices $\left(\mathrm{F} \propto \nu^{\alpha}\right)$ range from flat $(\alpha \sim 0)$ to steep $(\alpha \sim-1)$. The total $6 \mathrm{~cm}$ flux in Table 2 is given both for our images and for the single-dish observations by Wright et al. (1994, 1996). The difference between these indicates the amount of diffuse emission which is missing from our images. We also calculated the maximum brightness temperatures for each image (both 3 and $6 \mathrm{~cm}$ ) and the maximum value is given for each source.

In Table 3 we list various derived quantities for the sample including Hubble type, distance, inclination, $6 \mathrm{~cm}$ and $[\mathrm{FeII}]$ line luminosities and the SN rate. The [FeII] measurements are from Moorwood \& Oliva (1988) in a 6 arcsec diameter aperture, except for the Circinus galaxy in which we use a nuclear [FeII] flux from Davies et al. (1997). None of the [FeII] fluxes have been corrected for extinction. The SN rate is calculated separately from both the $6 \mathrm{~cm}$ and [FeII] line flux in the 6 arcsec aperture (corresponding to $500 \mathrm{pc}$ at a typical distance of $20 \mathrm{Mpc}$ ). The $6 \mathrm{~cm}$ SN rate is calculated assuming that all of the $6 \mathrm{~cm}$ flux is non-thermal emission from cosmic rays accelerated by SNRs (e.g. Condon \& Yin 1990). This may give an overestimate of the rate because it ignores any nuclear flux (which may be significant) and the contribution from thermal emission (which is unlikely to be significant). For the [FeII] SN rate we simply assume an average luminosity of $2 \times 10^{36} \mathrm{erg} \mathrm{s}^{-1}$ over an adiabatic lifetime of $2 \times 10^{4} \mathrm{yrs}$ (e.g. Norris \& Forbes 1995). For most galaxies the two methods give rates within a factor of two, the notable exception being NGC 4945 (which is discussed further below).

\subsection{Radio Spectral Indices}

The radio spectral index of Seyfert and starburst galaxies is determined by four mechanisms.

1. Cosmic rays within the galaxy are generated and re-accelerated by supernovae and supernova remnants, and then interact with the interstellar magnetic field to emit synchrotron emission. This synchrotron emission, with a typical spectral index of $\alpha \sim-0.7$, is expected to dominate the radio power of starburst galaxies, and should appear as a diffuse component in radio images of these sources.

2. Relativistic particles ejected from the massive black hole at the nucleus of a galaxy may generate intense synchrotron emission, similar to that seen in radio-loud galaxies and quasars. The cores in these radio-loud objects typically have a flat-spectrum core, indicating synchrotron self-absorption, and steep-spectrum extended radio-lobes, suggesting cooling of high-energy electrons. However, synchrotron self-absorption is important only for brightness temperatures greater than $10^{10} \mathrm{~K}$ (Condon 1992). Most Seyfert galaxies, on the other hand, are observed to have much lower brightness temperatures than this in the core, so that synchrotron self-absorption is not significant in these sources. This is confirmed by the observed core spectral indices, which are frequently in the region of -0.7 .

3. When the radiative efficiency in the accretion disk is low, an advection-dominated accretion flow (ADAF) may operate. The radio emission in these sources is dominated by synchrotron emission from a hot plasma, and the emission from such flows is predicted to give rise to inverted spectra with typical indices of +0.4 (Yi \& Boughn 1997). The ADAF radio emission mechanism has only recently been proposed 
and should be regarded as untested at this stage. The data here are unlikely to provide a definitive test because of insufficient resolution, and all radio spectral indices measured here are negative. We will therefore not consider this mechanism further, except to note that this mechanism would be indicated by inverted-spectrum emission from a low-brightness-temperature core. No source here falls into this category.

4. H II regions in our galaxy generate free-free emission from hot electrons. Most are optically thin, giving a flat spectrum, although some compact H II regions become optically thick at centimetre wavelengths, giving a spectral index $\sim+2$. However, the integrated flux of such regions is generally insignificant compared to the synchrotron emission of the host galaxy.

5. The radio emission from ultra-luminous infrared galaxies is optically thick to free-free absorption, so that the typical synchrotron spectrum of these galaxies is flattened at low frequencies (Condon et al. 1991).

The combined result of these effects in Seyfert and starburst galaxies is to produce a typical radio spectral index of -0.7 (from the extended synchrotron emission) with a flattening at low frequencies in some starburst sources because of free-free absorption.

Table 2 shows that the nuclear spectral indices of three of the galaxies (NGC 1365, NGC 6221, NGC 7582 ) is -0.5 or steeper on both the 2 arcsec and the 6 arcsec scale, showing evidence for neither free-free nor synchrotron absorption. In the other three galaxies, the cores have flatter spectra, but the brightness temperatures $(\leq 8300 \mathrm{~K})$ are too low for synchrotron self-absorption, indicating that free-free absorption is responsible for the flattening.

Of course, we cannot rule out the presence of a weak synchrotron self-absorbed core in the nucleus of any of these galaxies. However, comparison of the radio fluxes in a 2-arcsec aperture with the flux in a 6 -arcsec aperture in Table 2 shows that the luminosity of any such core is small compared to the surrounding emission. Therefore any such core does not contribute significantly to the overall energy budget of the nuclear region of the galaxy, and is not responsible for the overall flat spectrum of the nuclear region..

This degree of free-free absorption flattening indicates either a high star formation rate (Condon et al. 1991) or that we are viewing the AGN through optically-thick narrow-line-region clouds (Roy et al. 1994).

\subsection{The Radio - [FeII] Correlation}

Forbes \& Ward (1993) discovered that the $6 \mathrm{~cm}$ radio emission in the central regions of active galaxies is strongly correlated with the near-infrared [FeII] $1.64 \mu \mathrm{m}$ line emission. This relation exists over several orders of magnitude. With a larger sample, Simpson et al. (1996) were able to show that Seyfert and starburst galaxies follow slightly different radio-[FeII] relations. For starburst galaxies the relation, with slope $\sim 1$, can be reasonably explained by SNRs which are responsible for both the non-thermal radio emission and the fast shocks that provide the $[\mathrm{FeII}]$ excitation. However, the situation for Seyfert galaxies (which reveal a correlation slope of $\sim 0.7$ ) is less clear. Simpson et al. argued that photo-ionisation from the Seyfert nucleus can cause this relationship, with a contribution from radio-jet induced shocks in some cases.

In Fig. 13 we show the $[\mathrm{FeII}] / 6 \mathrm{~cm}$ ratio for our sample galaxies, compared with the Seyfert and starburst relations of Simpson et al. (1996). The $1 \sigma$ dispersion of galaxies about the relations is $\sim 10^{0.5}$. 
For the Seyferts studied here, we find a large degree of star-formation activity compared to photo-ionisation from an AGN, and so we might expect them to lie closer to the starburst relation than the Seyfert one. This indeed appears to be the case for four galaxies, although one (NGC 7582) is closer to the Seyfert relation and NGC 4945 falls well away from either relation. The [FeII] $6 \mathrm{~cm}$ ratio of NGC 4945 is about a factor of 100 lower than typical active galaxies, and we discuss this further in Section 3.4 below. We note however that given the dispersion in the relations, and the low luminosities $\left(\leq 10^{40} \mathrm{erg} \mathrm{s}^{-1}\right)$ of the galaxies studied here, this is not a sensitive test of the excitation mechanism.

\subsection{The Radio - FIR Correlation}

Normal spiral and starburst galaxies show a tight correlation between their radio and FIR luminosity (e.g. Wunderlich et al. 1987). This correlation, which extends over five orders of magnitude, is true for

both flux density and luminosity, and cannot be accounted for by selection effects, or by a simple "richness effect". While a detailed mechanism to explain this correlation has yet to be established, it is almost certainly the result of star formation, which generates both the synchrotron radio emission and the thermal FIR emission. This is supported by the fact that all objects that are dominated by star formation (HII galaxies, normal spirals, starburst galaxies) do follow the correlation.

On the other hand, Sopp \& Alexander (1991) showed that radio-loud quasars and radio galaxies clearly do not follow the radio-FIR correlation. Thus whether or not a galaxy follows this correlation may be used as an indicator of the dominant radio luminosity source of the galaxy.

Norris et al. (1988) and Roy et al. (1997) showed that Seyfert galaxies, unlike radio-loud quasars, do roughly follow the radio-FIR correlation, but with a looser fit than normal spirals and starbursts. This suggests that the bolometric luminosity of Seyfert galaxies may be dominated by star formation. This is supported by off-nuclear optical and infrared observations of Seyferts, which show the same line ratios and luminosities as starburst galaxies (Bransford et al. 1997). Thus, although the nuclear optical spectra of Seyfert galaxies are clearly dominated by an AGN, in many cases the integrated radio emission and the FIR emission are dominated not by the AGN but by circumnuclear star formation.

The degree to which an individual galaxy follows this correlation is most conveniently expressed by the parameter $\mathrm{q}$ - the logarithm of the FIR to radio ratio. The conventional definition of $\mathrm{q}$ follows that of Helou et al. (1985), who define q in terms of the $1.49 \mathrm{GHz}$ radio flux. For our purposes, we adapt Helou's definition to our observing frequency of $4.8 \mathrm{GHz}$ by assuming a spectral index of -0.7 , and therefore define it as

$$
\begin{aligned}
& \mathrm{q} \equiv \log \left[\left(\mathrm{FIR} / 3.75 \times 10^{12} \mathrm{~Hz}\right) /\left(2.26 \times \mathrm{S}_{4.8 \mathrm{GHz}}\right]\right. \\
& \text { where } \mathrm{FIR} \equiv 1.26 \times 10^{-14}\left(2.58 \mathrm{~S}_{60 \mu}+\mathrm{S}_{100 \mu}\right)
\end{aligned}
$$

Typical values of q from the IRAS Bright Galaxy Sample are 2.34 for normal spirals, 2.21 for starburst galaxies, and less than 2 for radio-loud AGNs (Condon et al. 1991).

All the galaxies studied here except NGC 4945 have q in the range 2.2 to 2.3 , which places them firmly in the middle of the radio-FIR correlation, and suggests that most of their radio and FIR luminosity is produced by star formation. We discuss the case of NGC $4945(\mathrm{q}=1.88)$ below. 


\subsection{Individual Galaxies}

Here we discuss each galaxy in turn, starting with an extended discussion of NGC 4945. To avoid repetition, we note that in every case other than NGC 4945, the spectral index, radio-FIR ratio, and [FeII]-radio ratio are all consistent with star formation, rather than an AGN, being the dominant source of radio emission.

NGC 4945 This infrared luminous galaxy is nearly edge-on and is located in a nearby loose group. Although we list it as a barred galaxy in Table 3, there is a continuing debate about the reality of the bar (e.g. Harnett et al. 1989). Koornneef (1993) described NGC 4945 as a post-starburst galaxy with no evidence for an AGN. However Moorwood \& Oliva (1994) have argued that the central regions do show signs of ongoing young star formation. Evidence for a heavily obscured AGN now come from the variable hard $\mathrm{X}$-rays (Iwasawa et al. 1993), and the presence of a compact radio core in VLBI observations (Sadler et al. 1995). The galaxy contains a thick torus or ring with a radius of $\sim 150 \mathrm{pc}$ (Koornneef 1993; Moorwood et al. 1996). Harnett et al. (1989) found that the radio emission has a strong central contribution with emission extended 10 arcmin perpendicular to the major axis. Multi-frequency observations have been carried out by Elmouttie et al. (1997). They focused on the large scale structure using a beam size of $\sim$ $25^{\prime \prime}$, and found that the spectral index steepens from the central region to the main disk of the galaxy.

Furthermore, NGC 4945 is notable for the fact that it is one of the few galaxies (along with Circinus) known to contain water megamasers. Such megamasers have been cited in NGC 4258 (Miyoshi et al. 1995) as the strongest evidence known for a massive black hole in an AGN. Preliminary VLBI imaging (Greenhill et al. 1997) of the megamasers supports the model that they are in a Keplerian disk surrounding the black hole.

Our radio image is dominated by strong nuclear emission, and emission extended along the disk of the galaxy. However there is also evidence of some filamentary structure perpendicular to the major axis. Such extended emission may be associated with the outflowing superwind in this galaxy (Nakai et al. 1989; Lipari, Tsvetanov \& Macchetto 1997). The extended emission has a steeper spectral index $(\alpha \sim-0.8)$ than the radio nucleus, however we note that our data are less sensitive to extended emission (particularly at

$3 \mathrm{~cm}$ ) which makes the spectral index somewhat less certain. The nucleus has a relatively flat spectral index of $\alpha=-0.3$. The brightness temperature in the central $2 \operatorname{arcsec}$ of this source (i.e. $7000 \mathrm{~K}$ ) is still far too low for synchrotron self-absorption, indicating that the star formation activity is particularly intense, to provide the necessary free-free absorption. The obscuration inferred from the $\mathrm{X}$-ray data suggest that the extinction towards the AGN could be as high as $\mathrm{A}_{V} \sim 2500$.

We noted above (in Section 3.3) that the radio-FIR ratio for this galaxy is unusually low (i.e. $\mathrm{q}=$ 1.88), which at first sight appears to suggest that an AGN is responsible for much of the radio emission. However, this galaxy is so near that not all the FIR flux was in the single IRAS aperture, and so the IRAS flux listed in the IRAS Point Source Catalog may be an underestimate. Rice et al. (1988) have estimated the total FIR flux by co-adding IRAS images and obtain a higher value for the FIR fluxes, which raises the value of q to 2.1, suggesting that the radio emission in this galaxy is again dominated by star formation rather than by an AGN.

An interesting property of NGC 4945 is that the ratio of the [FeII] line luminosity to $6 \mathrm{~cm}$ radio emission is only 0.63 , which is almost a factor of 100 less than is typical for active galaxies (see Fig. 13). We now consider a number of possible reasons for this. 
- The reduced ratio could be due to extinction (by dust) of the [FeII]. However, Moorwood \& Oliva (1994) estimate that the extinction in the [FeII] line emitting zone is 1.8 mag or a factor of five, which is insufficient to produce the observed effect.

- It could be because of nuclear radio emission from an AGN which is not accompanied by [FeII] line emission. We have shown above that the radio/FIR ratio for the galaxy as a whole is consistent with star formation activity. However, the central 6 arcsec (over which we measure the $[\mathrm{FeII}] / 6 \mathrm{~cm}$ ratio) contributes only $9 \%$ of the total radio flux, and we have no information on the radio/FIR ratio in the nucleus, so the radio flux from the AGN could be abnormally large. In this case, we would expect the $[\mathrm{FeII}] / 6 \mathrm{~cm}$ ratio to approach the usual value as we increase the area over which we integrate the flux (for both $6 \mathrm{~cm}$ and [FeII]). However, Moorwood \& Oliva (1994) quote a total [FeII] flux over an emitting region of $18 \times 21$ arcsec to be $12 \times 10^{-14} \mathrm{erg} \mathrm{s}^{-1} \mathrm{~cm}^{-2}$, or an observed log luminosity of $38.81 \mathrm{erg} \mathrm{s}^{-1}$. The $6 \mathrm{~cm}$ radio luminosity over a similar area is $40.21 \mathrm{erg} \mathrm{s}^{-1}$, giving a [FeII]/6cm ratio of 0.03 , which is even lower than the value in the nucleus, indicating that the $[\mathrm{FeII}] / 6 \mathrm{~cm}$ ratio falls off with distance from the galaxy centre, and that the low value is not a consequence of nuclear radio emission.

Thus the abnormally low [FeII] $/ 6 \mathrm{~cm}$ ratio in NGC 4945 of 0.63 is produced in the region surrounding the nucleus, where the radio emission (with a spectral index of $\sim-0.7$ ) is due to SNRs in the galaxy disk, the outflowing starburst superwind discussed above, or perhaps a radio jet. Pure SNRs produce ratios of about 500 i.e. well in excess of typical galaxy values, so this would tend to give an enhanced ratio. In a $6^{\prime \prime} \times 6^{\prime \prime}$ aperture, the superwind galaxies M82 and NGC 253 have [FeII] $/ 6 \mathrm{~cm}$ ratios of 75 and 52 respectively, although the superwind itself in NGC 253 does not seem to produce significant [FeII] line emission (Forbes et al. 1993). Again such ratios are significantly higher than seen in NGC 4945. The data for Seyfert galaxies with clear radio jets are limited. For NGC 4151 and NGC 1068 the measured ratios are 28 and 9 . This is closer to the NGC 4945 value but still a factor of at least 10 too high. We conclude that the abnormal [FeII] $/ 6 \mathrm{~cm}$ ratio in NGC 4945 is due to either (a) a starburst superwind, which produces substantial radio emission but very little [FeII] line flux (due perhaps to an unknown excitation effect or low density in the wind), or (b) a radio jet which dominates the extended radio emission on the few-arcsec scale but which does not produce significant [FeII] emission.

NGC 1365 This is a well-studied barred galaxy. The central region reveals broad and narrow emission lines (Veron et al. 1980) surrounded by a circumnuclear ring of star formation (Edmunds \& Pagel 1982; Saikia et al. 1994). The star formation, combined with the obscuring effects of dust, give the appearance of a hot-spot nucleus (Sersic \& Pastoriza 1965). A high excitation outflow from the nucleus has been seen (e.g. Hjelm \& Lindblad 1996).

High resolution radio continuum observations have been reported by several workers (e.g. Sandqvist, Jorsater \& Lindblad 1982, 1995). In particular, Sandqvist, Jorsater \& Lindblad (1995) observed it with the VLA at 20,6 , and $2 \mathrm{~cm}$. Their radio images revealed a weak nucleus surrounded by a elongated $\sim 8$ $\times 20 \operatorname{arcsec}(\mathrm{a} / \mathrm{b}=0.4)$ ring of hot-spots, or components. They labelled a number of components $\mathrm{A}-\mathrm{H}$, of which $\mathrm{B}$ is blended with $\mathrm{A}$ and $\mathrm{C}$ is blended with $\mathrm{D}$ at $\geq 1 \operatorname{arcsec}$ resolutions. Our radio image, shown in Fig. 1, is consistent with theirs, except that we identify one additional component to the SW, which we call ' $\mathrm{J}$ '. The hot-spots generally have steep spectra with $6 \mathrm{~cm}$ luminosities of $\sim 10^{36} \mathrm{erg} \mathrm{s}^{-1}$ which suggests that the radio emission from each component is made up of several SNRs. A combined radio and X-ray study of the nucleus and surrounding regions has been carried out by Stevens, Forbes \& Norris (1998). The radio nucleus does not appear to have an $\mathrm{X}$-ray counterpart. Furthermore the $\mathrm{X}$-ray emission is consistent 
with star formation processes. Stevens et al. conclude that if NGC 1365 harbours a black hole it is largely inactive.

NGC 6221 Located in a small group, NGC 6221, is a barred galaxy with a weak Seyfert nucleus. The galaxy may be interacting with NGC 6215 and has a nuclear bar (Koribalski 1996). The radio emission from NGC 6221 is extended in an symmetric bar-like structure. The spectral index of the nucleus and bar are non-thermal with $\alpha \sim-0.6$, indicative of SNRs. The radio morphology and other properties are all consistent with star formation being the dominant source of radio emission.

NGC 6810 This early type spiral is the most distant in our sample and has not been well studied to date. It may contain a bar and ring structure (Buta 1995), and does not appear to have been imaged before at radio wavelengths. Our radio image reveals a dominant nucleus surrounded by diffuse extended radio emission. Both the nucleus and surrounding region have flat spectral indices, but the brightness temperature is too low for this to be attributable to synchrotron self-absorption, which suggests that the radio spectrum is flattened by free-free absorption from young star formation. The radio morphology and other radio properties are all consistent with star formation being the dominant source of radio emission. Interestingly, recent high resolution optical spectra do not confirm the status of NGC 6810 as a Seyfert galaxy (Heisler 1998), thus it appears to have been misclassified.

NGC 7582 This narrow line X-ray galaxy is located in the Grus loose group along with NGC 7590 (a Seyfert 2; Ward et al. 1980), NGC 7552 (a starburst; Forbes et al. 1994) and NGC 7599. Several HI bridges connect group members (Koribalski 1996). Morris et al. (1985) provide evidence for both a rapidly-rotating $\sim 1 \mathrm{kpc}$ ring of circumnuclear star formation and high excitation gas moving outwards from the nucleus.

Ulvestad \& Wilson (1984) imaged the galaxy at $6 \mathrm{~cm}$ using the VLA with a beam size of $\sim 1.5$ arcsec. They measured a total $6 \mathrm{~cm}$ flux of $69 \mathrm{mJy}$. We find a linear, double-peaked morphology to the radio emission. The southern peak appears to lie at the centre of the outer radio isophotes and is presumably the true nucleus. The nucleus has a steep spectral index $(\alpha=-0.7)$ indicating non-thermal emission. To the NW by $\sim 3$ arcsec lies a second peak, which could be a second nucleus. However, it lies roughly along the bar/major axis position angle (P.A. $\sim 150^{\circ}$ ). It has a 2 arcsec diameter $6 \mathrm{~cm}$ flux of 10 mJy and a spectral index of -0.9 . This second peak could therefore be a radio jet or simply a discrete star formation region occurring along the galaxy bar. The inferred SN rate in the central $6 \operatorname{arcsec}(870 \mathrm{pc})$ is the highest in our sample (except possibly for NGC 4945) at about 1 SN every 8 years. Although the radio morphology suggests a linear Seyfert jet, the spectral index, radio-FIR ratio, and [FeII]-radio ratio are all consistent with star formation being the dominant source of radio emission.

Circinus The Circinus galaxy is perhaps the closest Seyfert galaxy known but is difficult to observe due to its proximity to the Galactic plane and large internal obscuration. Confirmation of an AGN comes from the the presence of high excitation coronal lines (Oliva et al. 1994), X-ray emission (Matt et al. 1996), and a compact radio core (Heisler et al. 1998).

Like NGC 4945, Circinus is one of the few water megamaser galaxies. The megamasers in Circinus are stronger but less extreme than those in NGC 4258, and have the curious property of fluctuating on a time scale of minutes (Greenhill et al. 1997), indicating a particularly compact source. Preliminary VLBI imaging of the megamasers (Ellingsen et al. 1998) indicates that the maser region is extended with a velocity gradient aligned with that of the parent galaxy, and perpendicular to the jet. We regard this as strong evidence for a massive black hole in this galaxy.

Marconi et al. (1994) found both a circumnuclear starburst ring and an ionisation cone. The [OIII] 
ionisation cone is asymmetric extending only to the NW, with some high excitation lines also seen in the cone region. They estimated the extinction to the nucleus to be $A_{V} \sim 20$. The HI gas distribution shows spiral arms, a bar and a central 'hole' (Koribalski 1996, Jones et al. 1998). High resolution observations of the central region indicate a rapidly-rotating gas ring with a diameter of $\sim 400 \mathrm{pc}$ (Koribalski 1996). Observations with the ATCA have been carried out at 13 and $20 \mathrm{~cm}$ by Elmouttie et al. (1995). They found extended radio lobes perpendicular to the galaxy major axis (position angle $=30^{\circ}$ ) with a spectral index of $\alpha \sim-0.7$.

Our 3 and $6 \mathrm{~cm}$ radio images, observed as part of this project, have been published, along with near-infrared line images, by Davies et al. (1997). The radio data indicate that the nucleus is marginally resolved with a flat spectral index. The low brightness temperature indicates that this is due to free-free absorption rather than synchrotron self-absorption in a compact AGN source. There are also faint hints of extended emission which may be associated with outflowing material. Despite the clear indication of a compact AGN in the radio images, the other radio indicators are consistent with the more extended radio emission being dominated by star formation activity.

\section{Summary and Conclusions}

We have imaged six southern active galaxies at both 3 and $6 \mathrm{~cm}$ using the ATCA. The radio emission reveals a variety of morphologies, from compact nuclear emission to extended diffuse structures. Several galaxies show radio components and linear features from the nucleus. Four galaxies (NGC 4945, 6810, 7582 and Circinus) reveal strong, fairly compact nuclear radio sources which may be due to synchrotron emission associated with an AGN, although the nuclear sources are not sufficiently compact for synchrotron self-absorption to be important. Three galaxies (NGC 1365, 7582 and Circinus) contain hints of jet-like features, but none are very convincing. NGC 1365 contains a very weak, compact nuclear radio source for which there is little evidence at radio or X-ray (Stevens et al. 1998) wavelengths for an AGN. NGC 6221 shows no evidence in the radio for the presence of an active nucleus, and in the case of NGC 6810 there are doubts about its optical classification as a Seyfert (Heisler 1998).

For most of the galaxies the SN rate, in the central $\sim 500 \mathrm{pc}$, inferred from [FeII] line luminosities are similar to those from $6 \mathrm{~cm}$ luminosities, suggesting that the extended non-thermal radio emission is dominated by diffuse synchrotron emission from cosmic rays accelerated by SNRs. In NGC 1365, the circumnuclear radio components are dominated by collections of SNRs which are more intense at radio wavelengths than the nucleus itself. For NGC 4945 additional radio emission appears to be coming from an outflowing superwind, perpendicular to the galaxy disk.

In all six galaxies the radio emission is very much dominated by processes associated with star formation. The AGN, if present, makes only a small energetic contribution, and we are unable to detect the presence of any advection-dominated accretion flows.

\section{Acknowledgments}

The Australia Telescope is funded by the Commonwealth of Australia for operation as a National Facility managed by CSIRO. We thank B. Koribalski and I. Stevens for their comments on the manuscript. We also thank the referee T. Muxlow for several useful suggestions. 


\section{References}

Antonucci, R. R. J., Ulvestad, J. S., 1988, ApJ, 330, L97

Bransford, M.A., Appleton, P.N, Heisler, C.A., Norris, R.P., Marston, A.P., 1997, ApJ, submitted

Buta, R., 1995, ApJS, 96, 39

Carral, P., Turner, J., Ho, P. 1990, ApJ, 362, 434

Collison, P. M., et al. 1994, MNRAS, 268, 203

Cid Fernandes, R., Terlevich, R., 1992, Relationships between Active Galactic Nuclei and Starburst

Galaxies, ed. A. V. Filippenko, ASP: San Francisco, p. 241

Condon, J. J., 1992, ARAA, 30, 575

Condon, J. J., Yin, Q. F., 1990, ApJ, 357, 97

Condon, J. J., Huang, Z.P., Yin, Q.F., Thuan, T.X., 1991, ApJ, 378, 65

Davies, R. I., et al. 1997, MNRAS, in press

Edmunds, M. G., Pagel, B. E. J., 1982, MNRAS, 198, 1089

Ellingsen, S., et al. 1998, in preparation

Elmouttie, M., et al. 1995, MNRAS, 275, 53

Elmouttie, M., et al. 1997, MNRAS, 284, 830

Filippenko, A. V., 1992, Relationships between Active Galactic Nuclei and Starburst Galaxies, ed. A. V. Filippenko, ASP: San Francisco

Forbes, D. A., Ward, M. J., 1993, ApJ, 416, 150

Forbes, D. A., Norris, R. P., Williger, G. M., Smith, R. C., 1994, AJ, 107, 984

Forbes, D. A., et al. 1993, ApJ, 406, L11

Greenhill, L. J., Moran, J. M., Herrnstein, J. R., 1997, ApJ, 481, 23

Harnett, J. J., Haynes, R. F., Klein, U., Wielebinski, R., 1989, A \& A, 216, 39

Heisler, C. 1998, personal communication

Heisler, C., et al. 1998, in preparation

Helou, G., Soifer, B. T., Rowan-Robinson, M., 1985, ApJ, 298, L7.

Hjelm, M., Lindblad, P. O., 1996, A \& A, 305, 727

Iwasawa, K., et al. 1993, ApJ, 409, 155

Jones, K., et al. 1998, MNRAS, in press

Koorneff, J., 1993, ApJ, 403, 581

Koribalski, B., 1996, Minnesota Lectures on Extragalactic Neutral Hydrogen ed. E. Skillman, ASP Conf.

Series 106, p 238

Kornberg, P. P., Biermann, P., Schwab, F. R., 1985, ApJ, 291, 693

Lipari, S., Tsvetanov, Z., \& Macchetto, F. 1997, ApJS, 111, 369

Marconi, A., Moorwood, A. F. M., Origlia, L., Oliva, E., 1994, The Messenger, 78, 20

Matt, G., et al. 1996, MNRAS, 281, 69

Miyoshi, M., Moran, J., Herrnstein, J., Greenhill, L., Nakai, N., Diamond, P., Inoue, M., 1995, Nature, 373, 127

Moorwood, A. F. M., Oliva, E., 1988, A \& A, 203, 278

Moorwood, A. F. M., Oliva, E., 1994, ApJ, 429, 602

Moorwood, A. F. M., van der Werf, P. P., Kotilainen, J., Marconi, A., Oliva, E., 1996, A \& A, 308, 1

Morris, S. L., et al. 1985, MNRAS, 216, 193

Nakai, N. 1989, PASJ, 41, 1107

Norman, C., Scoville, N., 1988, ApJ, 332, 124

Norris, R. P., Forbes, D. A., 1995, ApJ, 446, 594

Norris, R. P., Allen, D. A., Roche, P. F., 1988, MNRAS, 234, 773 
Oliva, E., et al. 1994, A \& A, 288, 457

Rice, W., Lonsdale, C.J., Soifer, B.T., Neugebauer, G., Kopan, E.L., Lloyd, A.L., de Jong, T., \& Habing, H.J., 1988, ApJS, 68, 91.

Roy, A. L., Norris, R. P., Kesteven, M. J., Troup, E. R., Reynolds, J. E., 1997, MNRAS, submitted Sadler, E., et al. 1995, MNRAS, 276, 1373

Saikia, D. J., Pedlar, A., Unger, S. W., Axon, D. J., 1994, MNRAS, 270, 465

Sanders, R. H., Bania, T. M., 1976, ApJ, 204, 341

Sandqvist, A., Jorsater, S., Lindblad, P. O., 1982, A \& A, 110, 336

Sandqvist, A., Jorsater, S., Lindblad, P. O., 1995, A \& A, 295, 585

Sersic, J. L., Pastoriza, M., 1965, PASP, 77, 287

Simpson, C., Forbes, D. A., Baker, A. C., Ward, M. J., 1996, MNRAS 283, 777

Sopp, H. M., Alexander, P. 1991, MNRAS, 251, 14P

Stevens, I. R., Forbes, D. A., Norris, R. P. 1998, in preparation

Ulvestad, J. S., Wilson, A. S., 1984, ApJ, 278, 544

Ulvestad, J. S., Wilson, A. S., 1984, ApJ, 285, 439

Veron, P., et al. 1980, A \& A, 87, 245

Ward, M. J., Penston, M. V., Blades, J. C., Turtle, A. J., 1980, MNRAS, 193, 563

Wright, A. E., Griffith, M. R., Burke, B. F., Ekers, R. D., 1994, ApJS, 91, 111

Wright, A. E., Griffith, M. R., Hunt, A. J., Troup, E., Burke, B. F., Ekers, R. D., 1996, ApJS, 103, 145

Wunderlich, E., Klein, U., Wielebinski, R., 1987, A \& AS, 69, 487

E. Skillman

Yi, I., Boughn, P., 1997, preprint (astro-ph/9710147) 


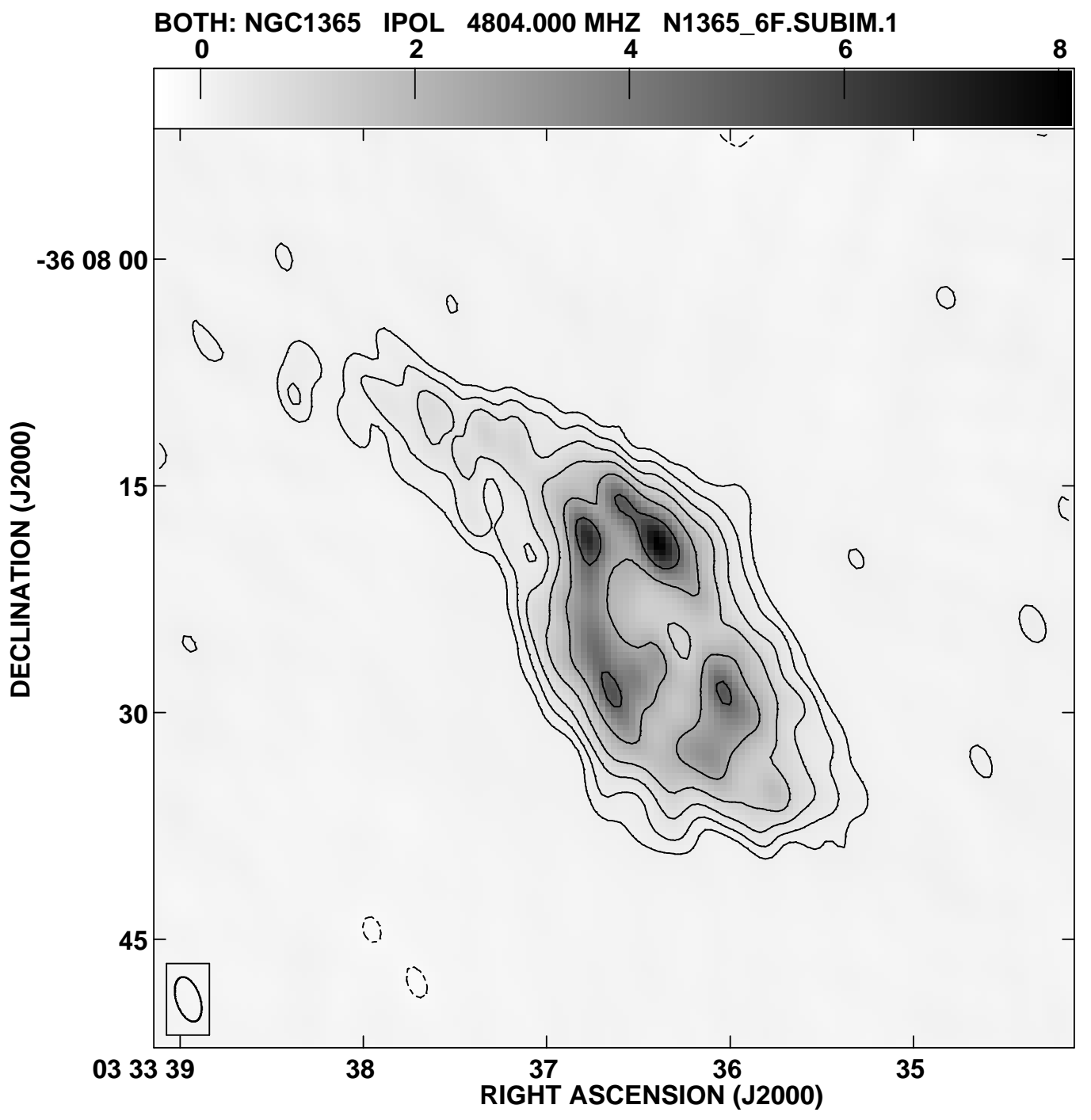

Fig. 1.- ATCA $6 \mathrm{~cm}$ image of NGC 1365 showing a ring of hot-spots surrounding a weak nucleus. In this and all subsequent figures, the horizontal grey-scale bar above the image shows the grey-scale in units of mJy per beam, and contours are at $-1,1,2,4,8,16,32$, and 64 times the lowest contour level. In this case the lowest contour level is $0.15 \mathrm{mJy} /$ beam. 


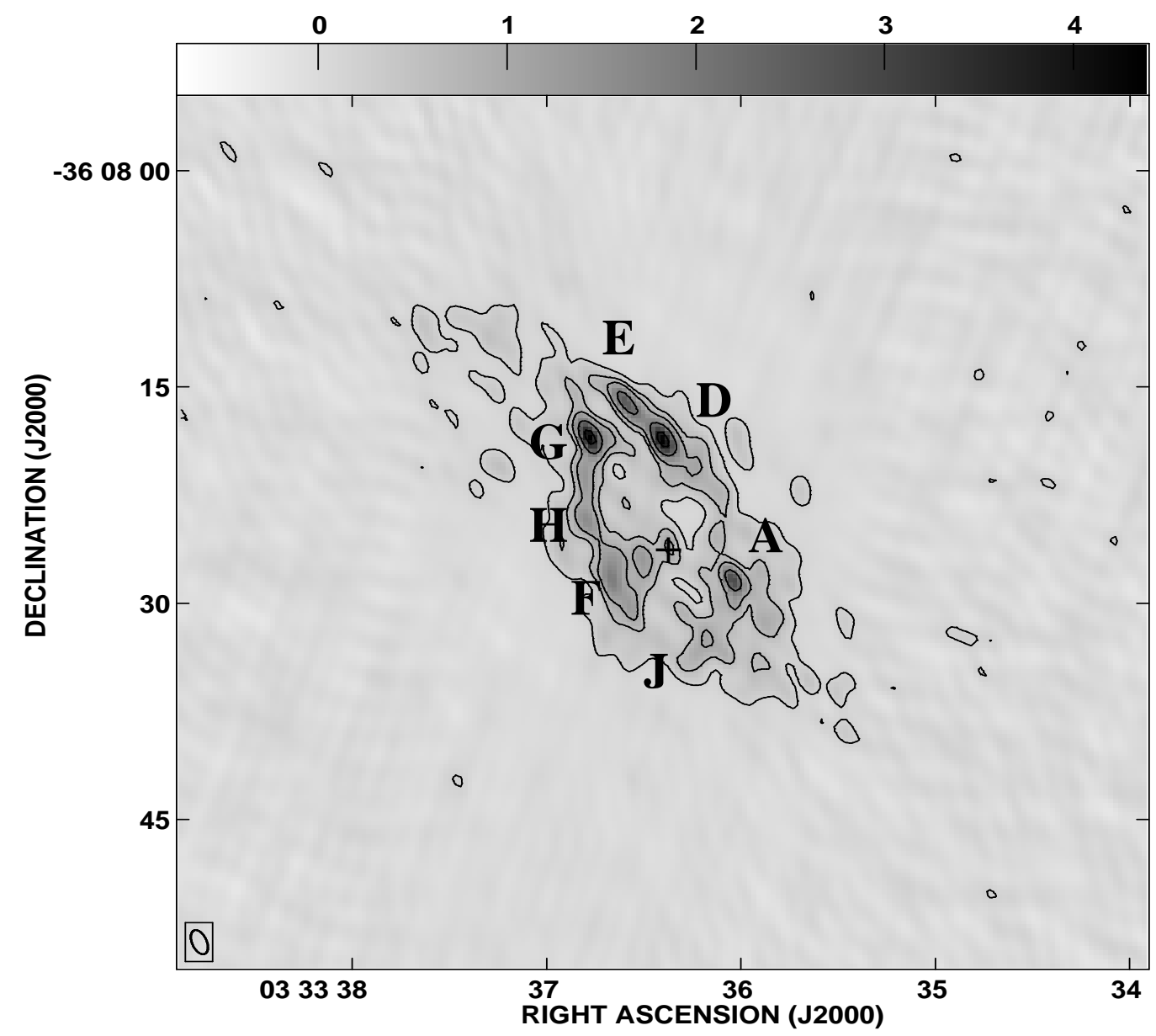

Fig. 2.- ATCA 3cm image of NGC 1365. The lowest contour level is $0.2 \mathrm{mJy} /$ beam. 


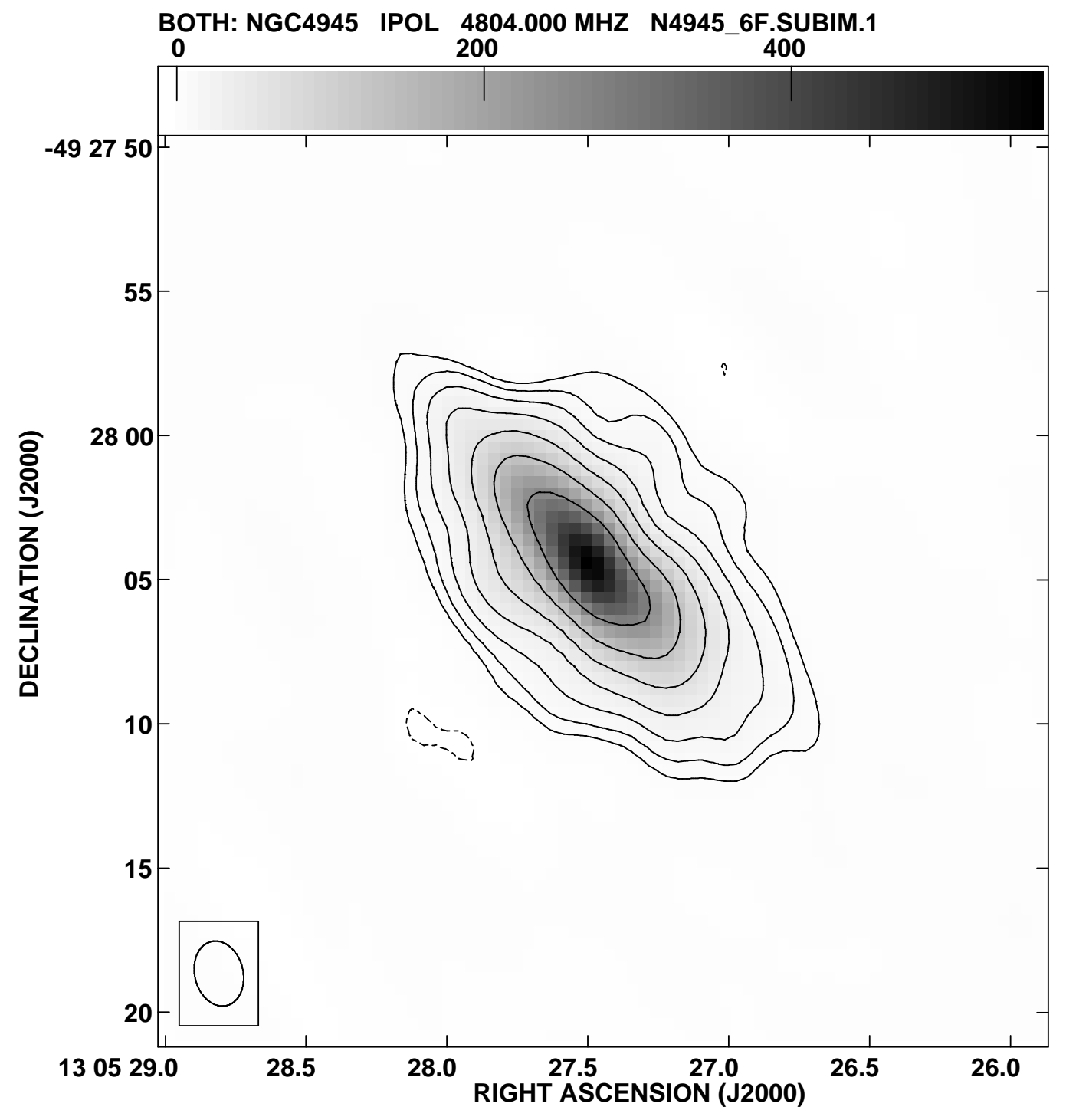

Fig. 3.- ATCA $6 \mathrm{~cm}$ image of NGC 4945 showing radio emission extended along the galaxy disk with some emission perpendicular to the plane. The lowest contour level is $4 \mathrm{mJy} /$ beam. 


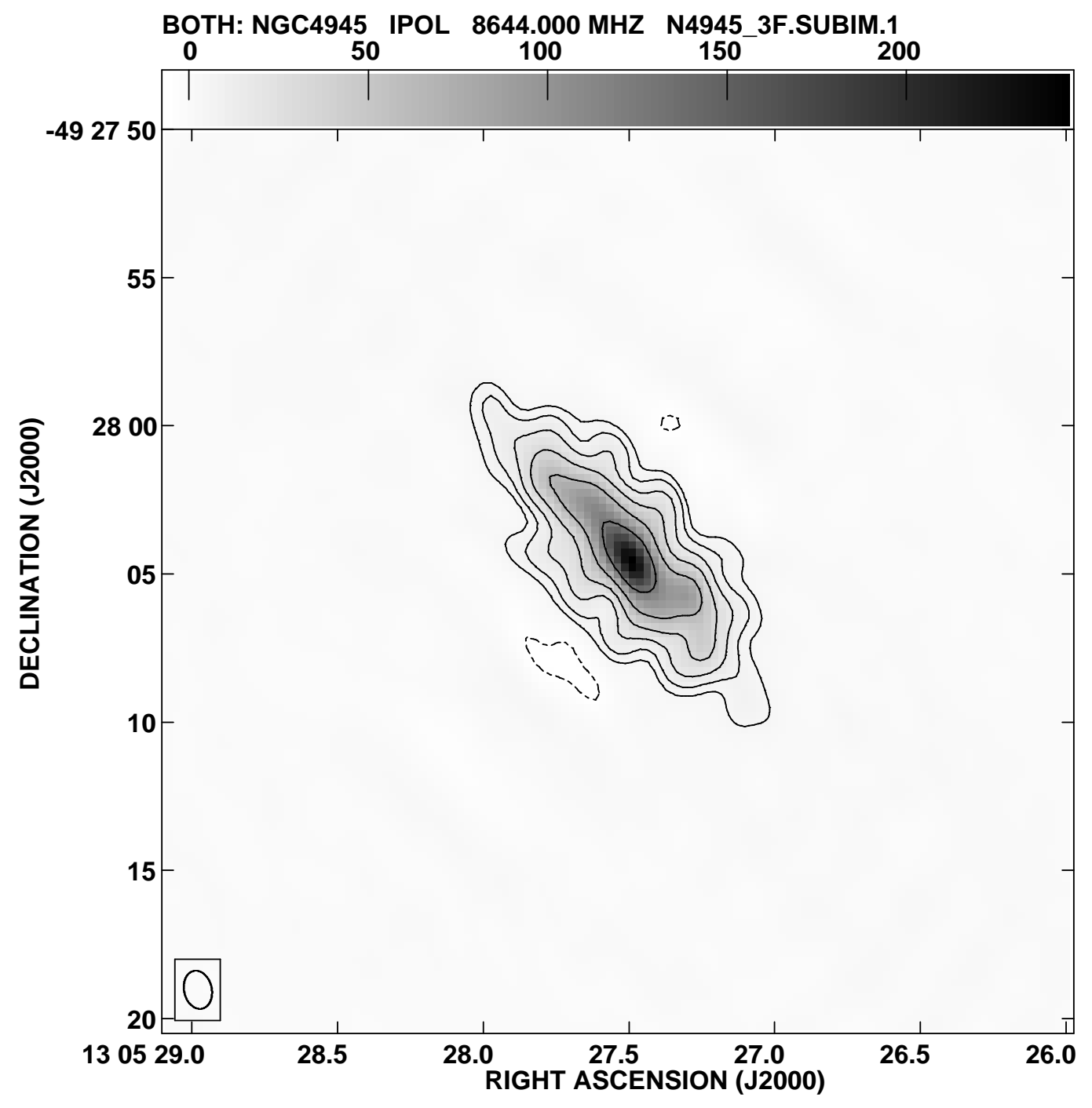

Fig. 4.- ATCA 3cm image of NGC 4945. The lowest contour level is $4 \mathrm{mJy} /$ beam. 


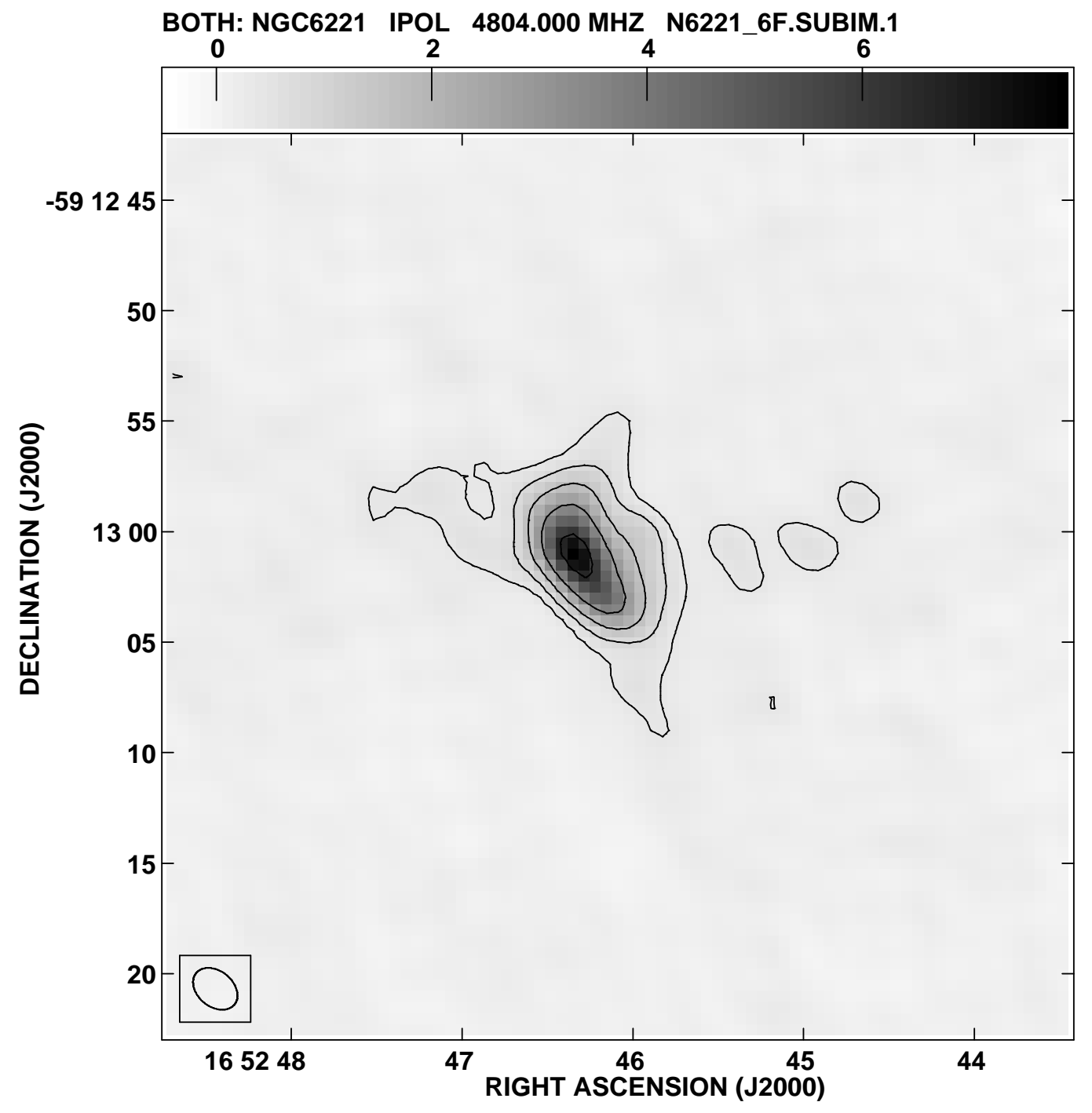

Fig. 5.- ATCA $6 \mathrm{~cm}$ image of NGC 6221 showing radio emission slightly extended beyond the nucleus. The lowest contour level is $0.4 \mathrm{mJy} /$ beam. 


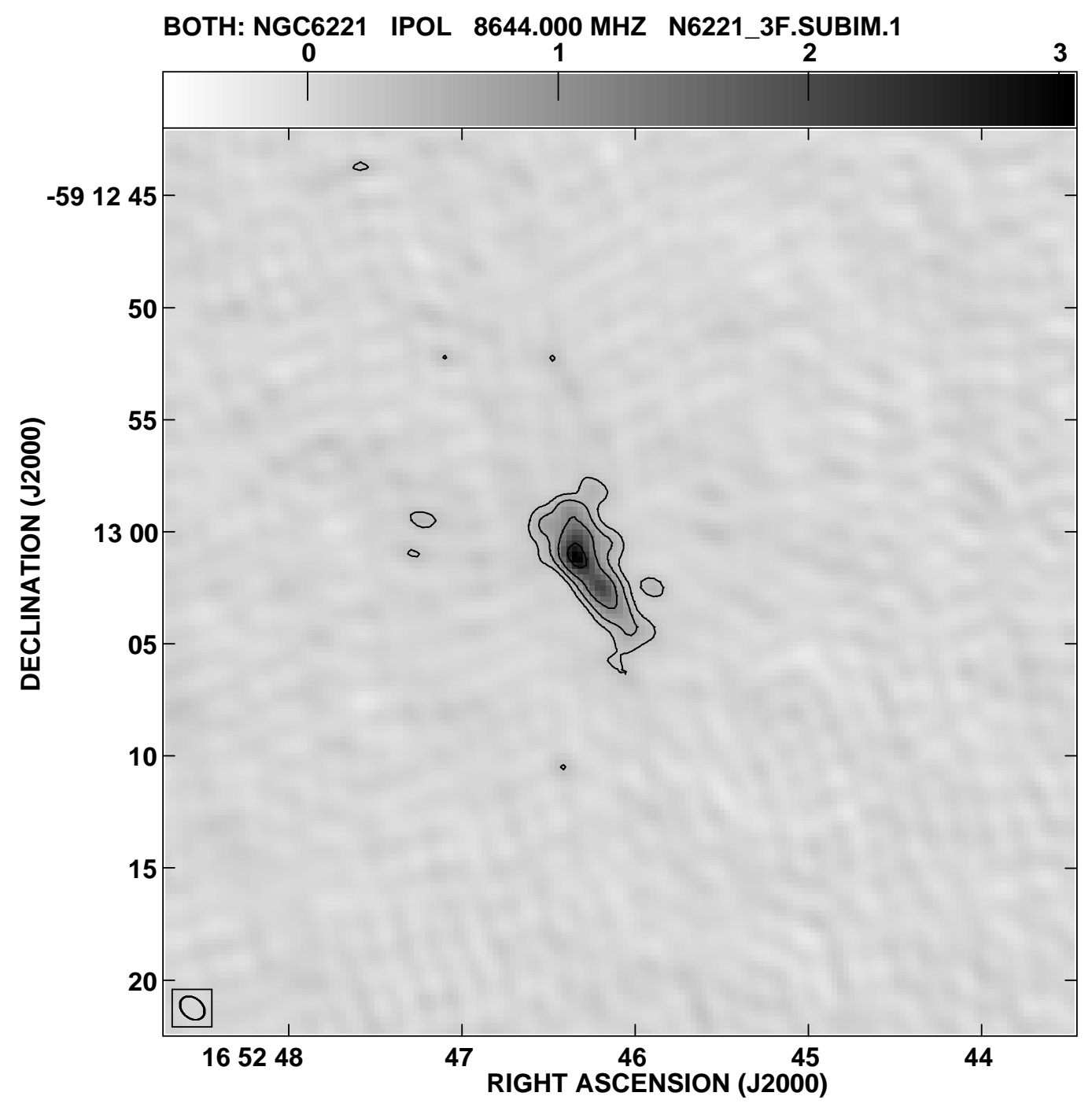

Fig. 6. - ATCA 3cm image of NGC 6221 . The lowest contour level is $0.3 \mathrm{mJy} /$ beam. 


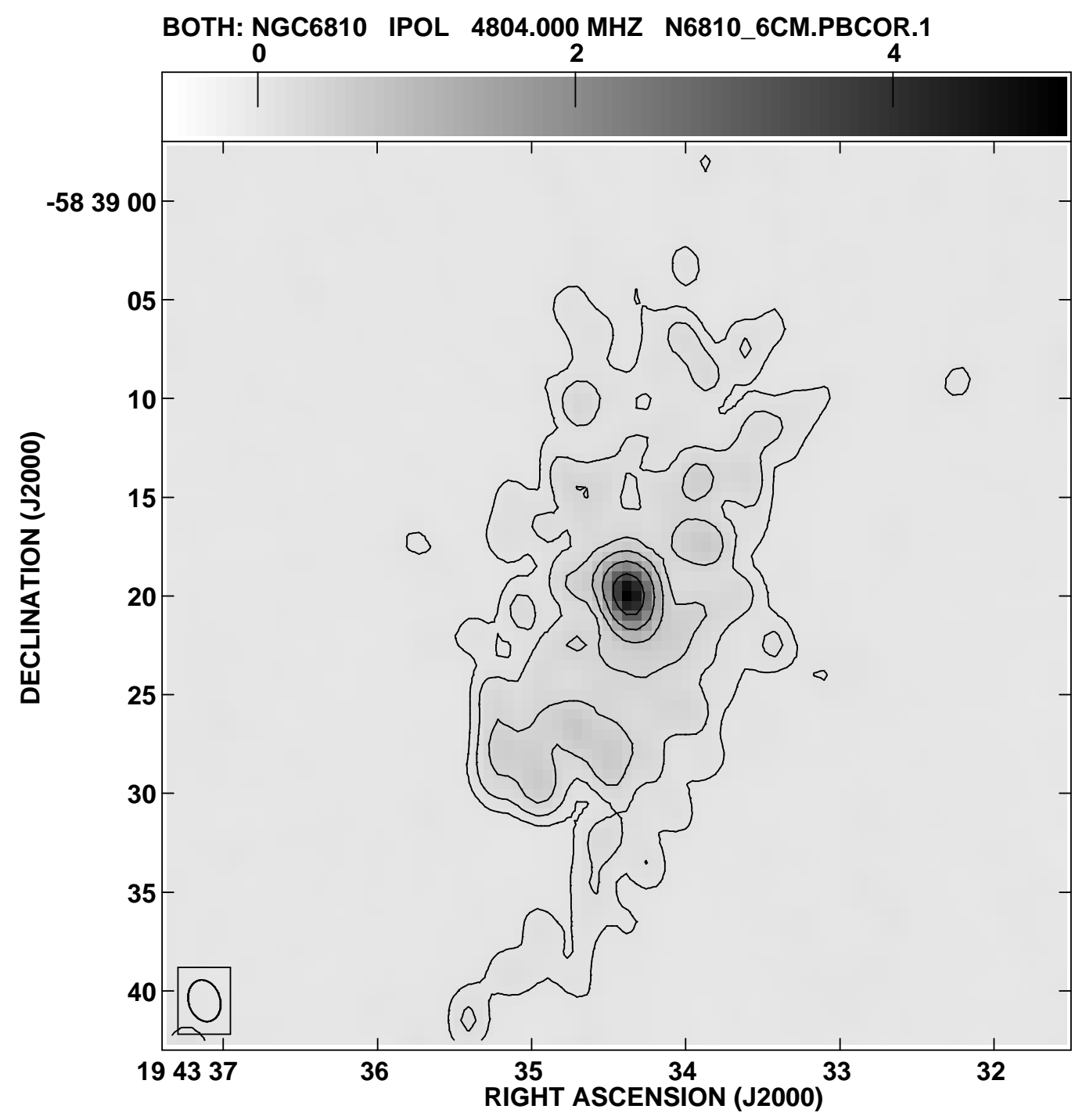

Fig. 7.- ATCA $6 \mathrm{~cm}$ image of NGC 6810 showing a compact nuclear source surrounded by weak emission. The lowest contour level is $0.1 \mathrm{mJy} /$ beam. 


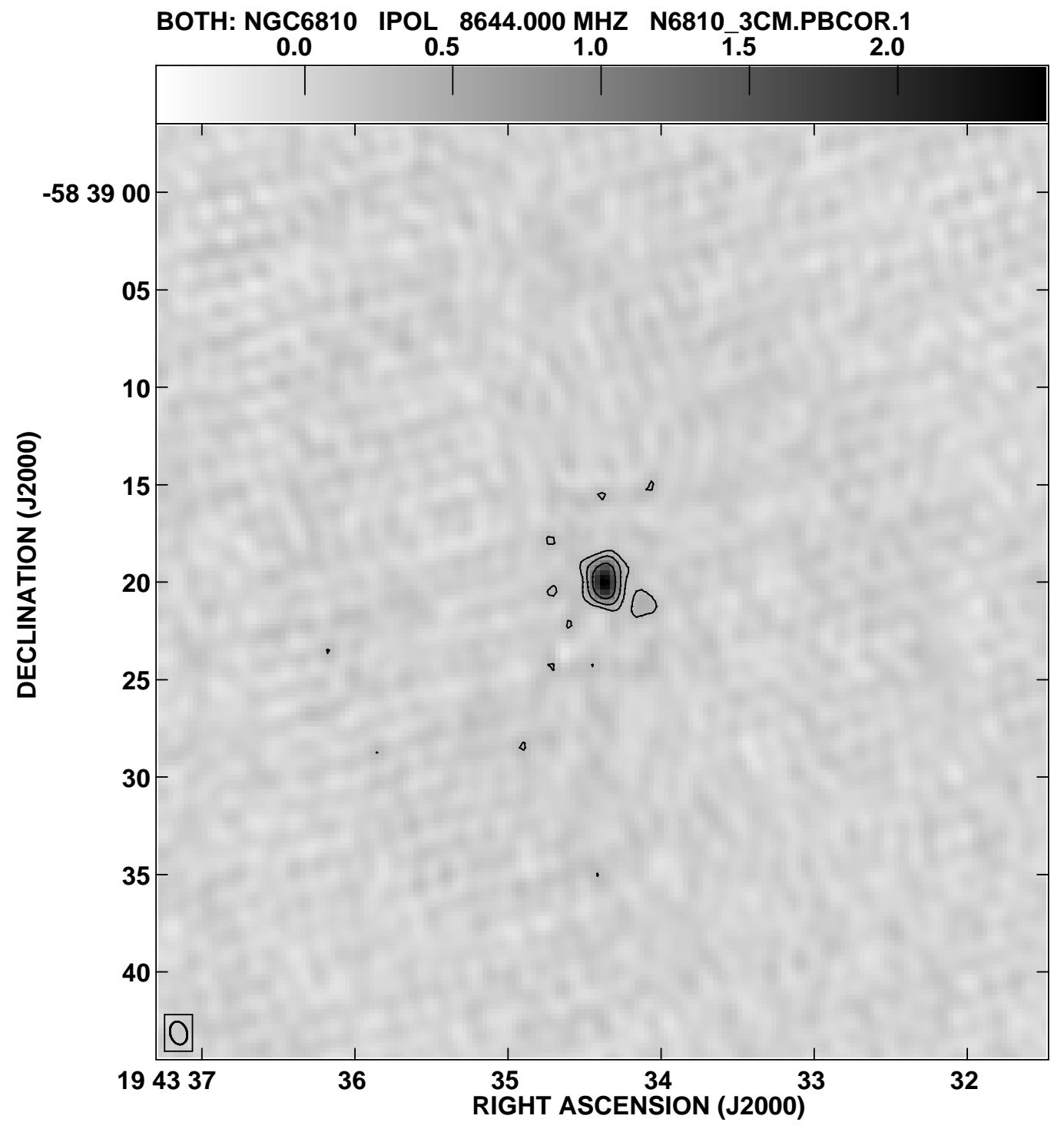

Fig. 8.- ATCA 3cm image of NGC 6810 . The lowest contour level is $0.3 \mathrm{mJy} /$ beam. 


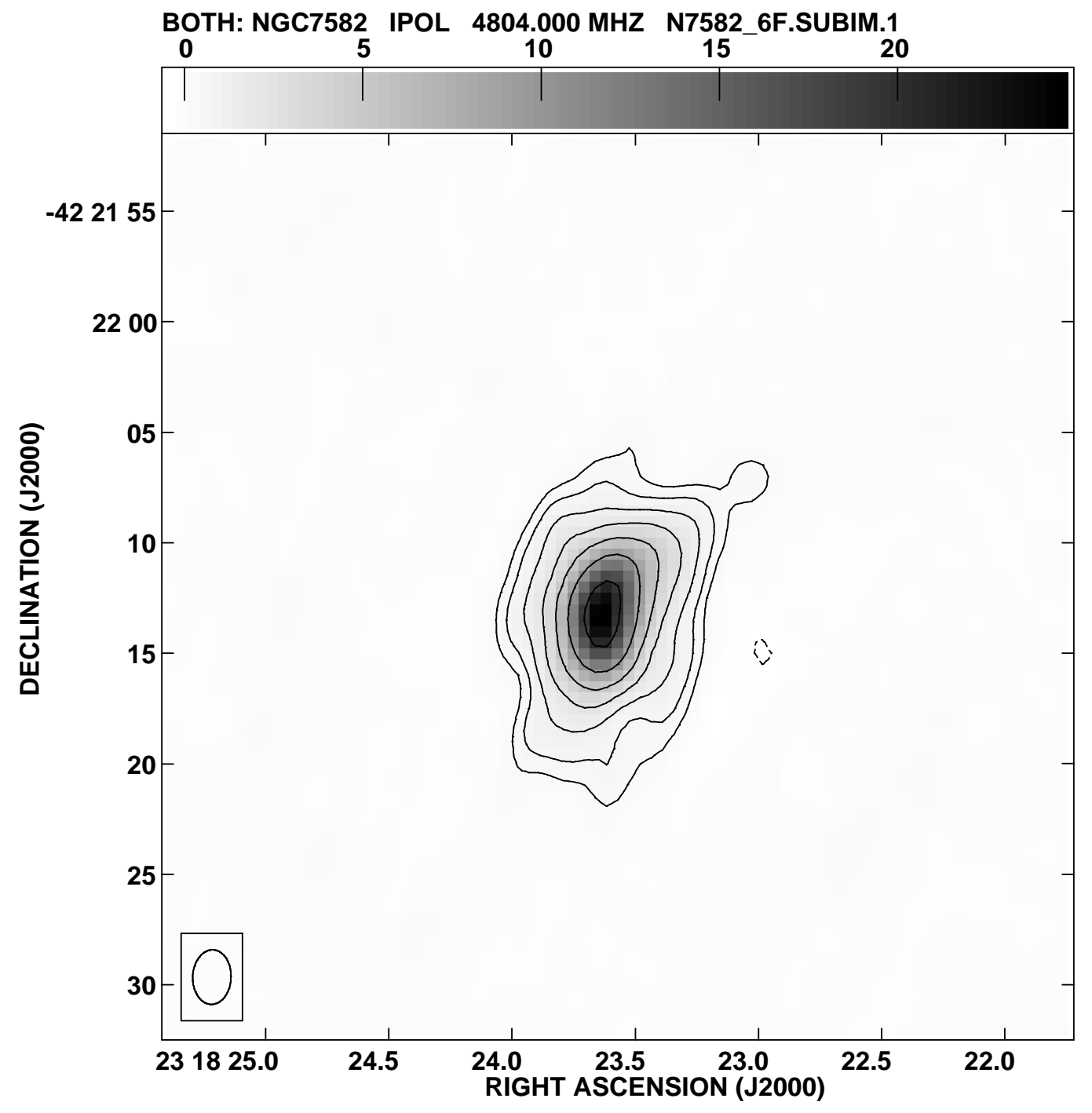

Fig. 9.- ATCA $6 \mathrm{~cm}$ image of NGC 7582 showing the nucleus and elongated extended emission. The lowest contour level is $0.2 \mathrm{mJy} /$ beam. 


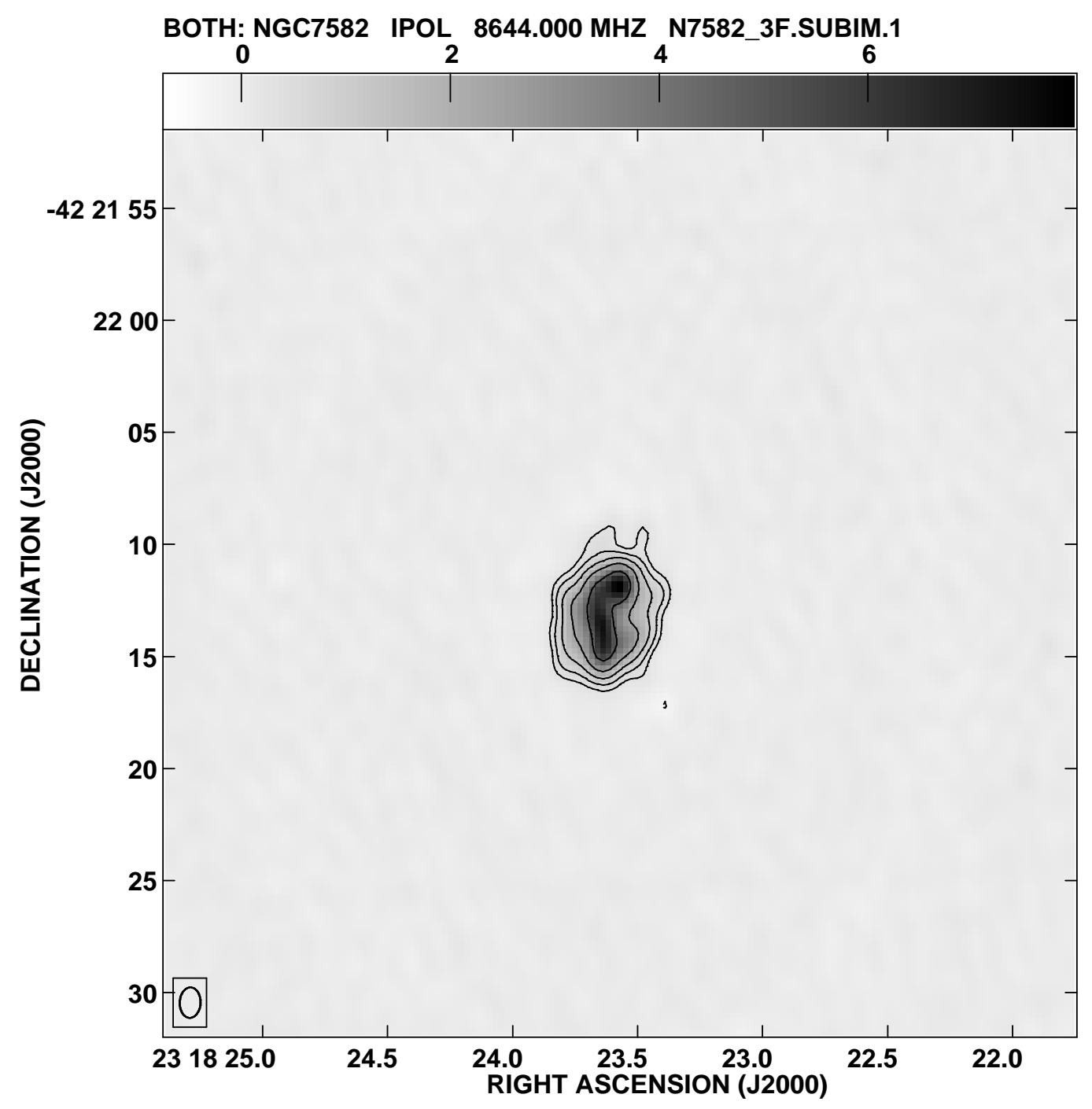

Fig. 10. - ATCA 3cm image of NGC7582. The lowest contour level is $0.2 \mathrm{mJy} /$ beam. 


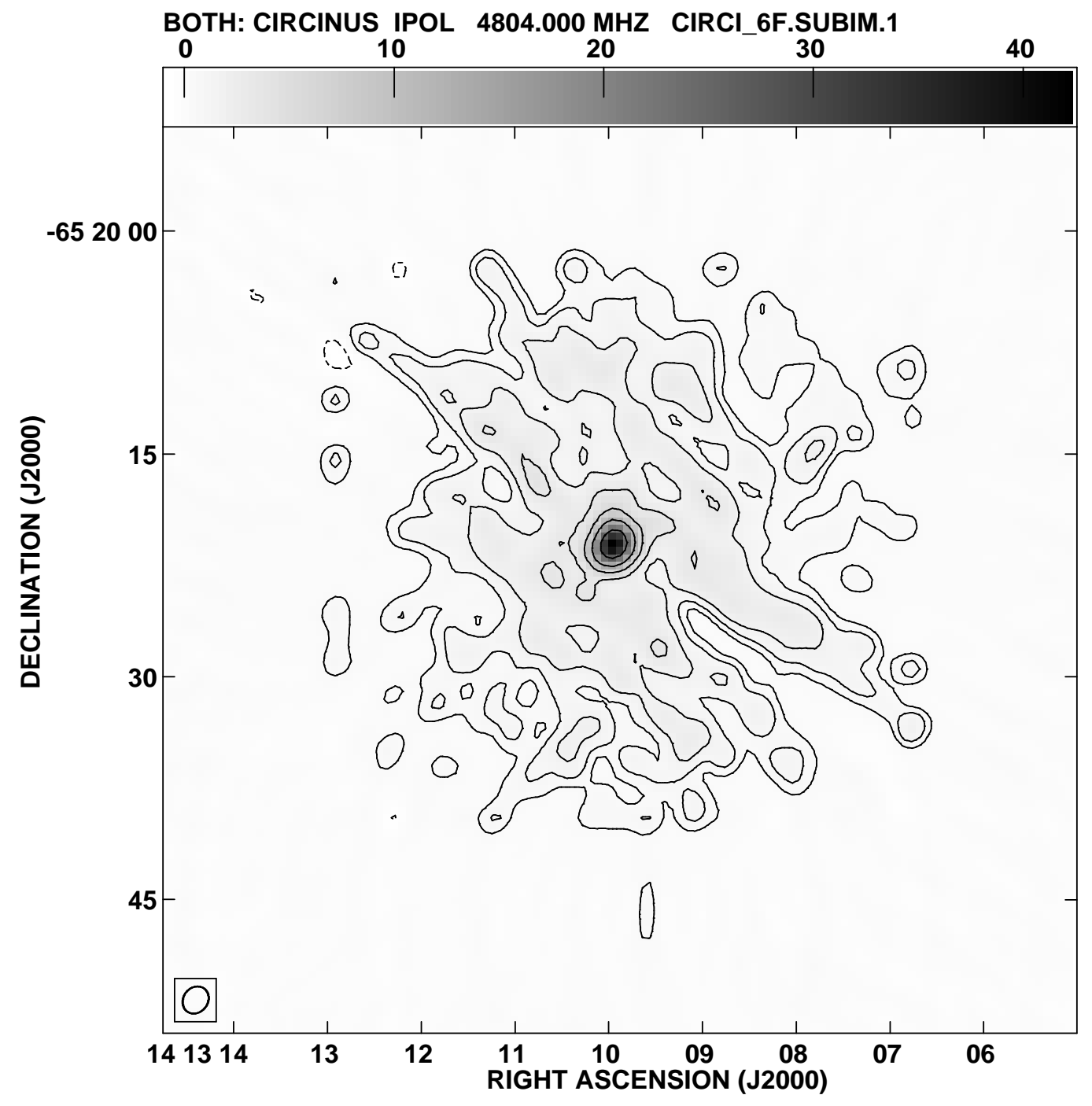

Fig. 11.- ATCA $6 \mathrm{~cm}$ image of Circinus showing a compact nuclear source and weak extended emission. The lowest contour level is $0.4 \mathrm{mJy} /$ beam. 


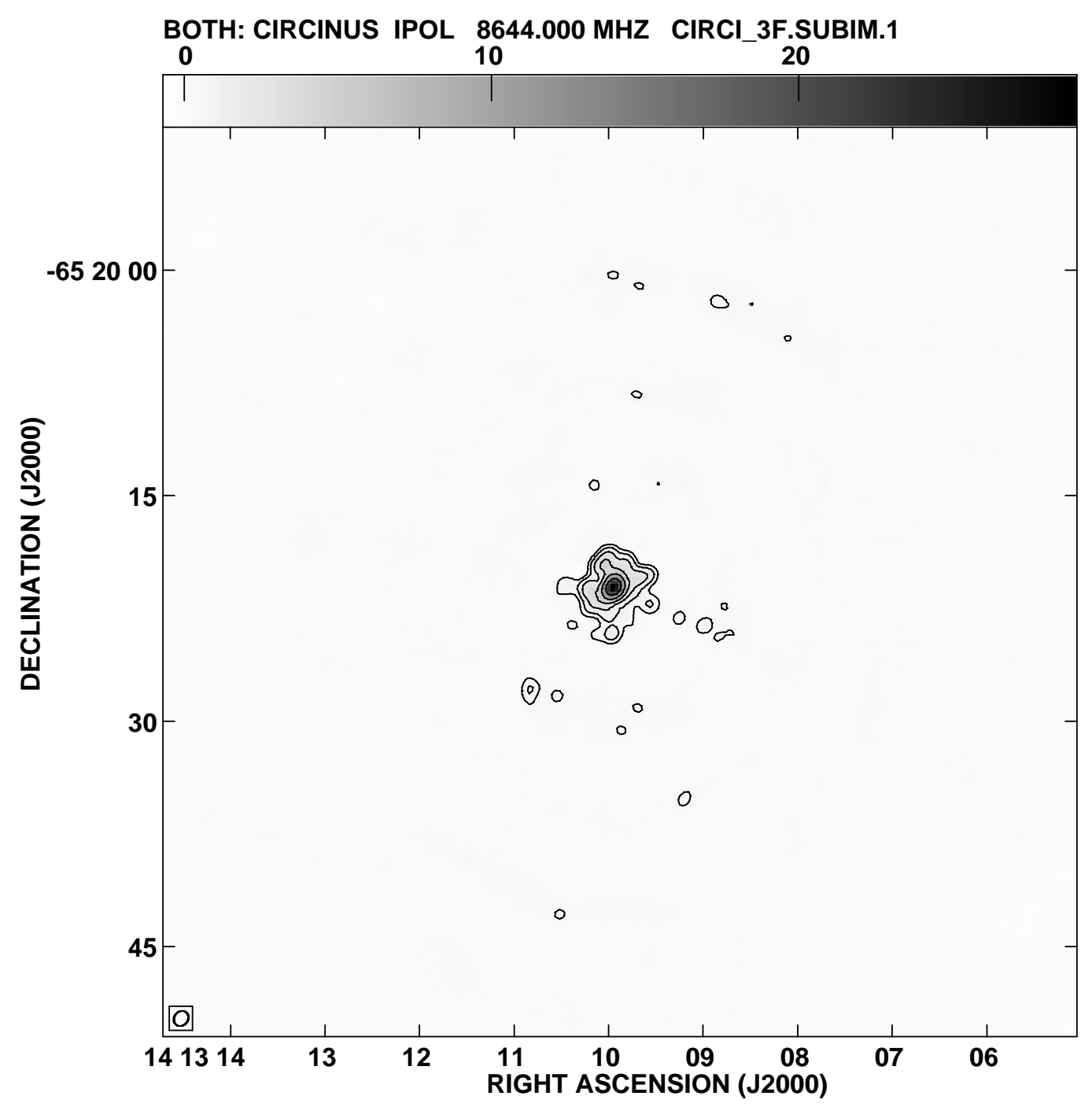

Fig. 12. - ATCA 3cm image of Circinus. The lowest contour level is $0.5 \mathrm{mJy} /$ beam. 


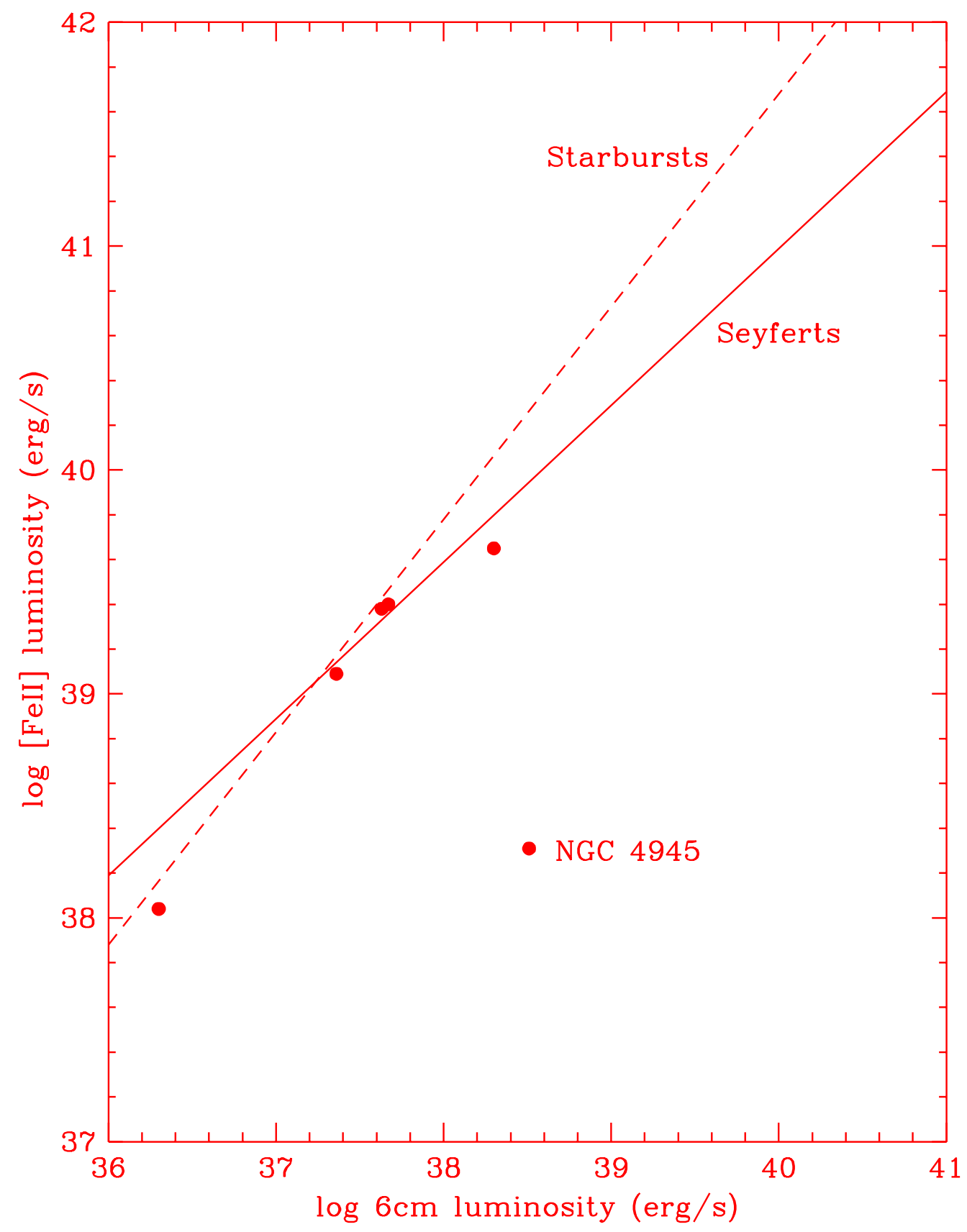

Fig. 13.- The $[\mathrm{FeII}]-6 \mathrm{~cm}$ radio correlation. The dashed line represents starburst galaxies and the solid line Seyferts, from Simpson et al. (1996). The dispersion about each line is $\sim 0.5$ in the log. Our sample galaxies are shown as filled circles. 
TABLE 1. Observations

\begin{tabular}{lcccccccc}
\hline \hline Galaxy & Date & $\begin{array}{c}\text { Phase } \\
\text { Calibrator }\end{array}$ & $\begin{array}{c}3 \mathrm{~cm} \\
\text { Beam }\end{array}$ & $\begin{array}{c}3 \mathrm{~cm} \\
\text { P.A. }\end{array}$ & $\begin{array}{c}3 \mathrm{~cm} \\
\mathrm{rms}\end{array}$ & $\begin{array}{c}6 \mathrm{~cm} \\
\text { Beam }\end{array}$ & $\begin{array}{c}6 \mathrm{~cm} \\
\text { P.A. }\end{array}$ & $\begin{array}{c}6 \mathrm{~cm} \\
\mathrm{rms}\end{array}$ \\
\hline \multirow{2}{*}{ NGC 1365 } & 1994 Nov. 10 & $0400-319$ & $1.76^{\prime \prime} \times 0.91^{\prime \prime}$ & $21^{\circ}$ & $50 \mu \mathrm{Jy}$ & $3.06^{\prime \prime} \times 1.57^{\prime \prime}$ & $18^{\circ}$ & $40 \mu \mathrm{Jy}$ \\
NGC 4945 & 1993 Nov. 19 & $1320-446$ & $1.30^{\prime \prime} \times 0.93^{\prime \prime}$ & $17^{\circ}$ & $500 \mu \mathrm{Jy}$ & $2.28^{\prime \prime} \times 1.67^{\prime \prime}$ & $13^{\circ}$ & $600 \mu \mathrm{Jy}$ \\
NGC 6221 & 1994 Nov. 13 & $1718-649$ & $1.21^{\prime \prime} \times 0.90^{\prime \prime}$ & $50^{\circ}$ & $45 \mu \mathrm{Jy}$ & $2.23^{\prime \prime} \times 1.60^{\prime \prime}$ & $50^{\circ}$ & $40 \mu \mathrm{Jy}$ \\
NGC 6810 & 1994 Nov. 17 & $1950-613$ & $1.20^{\prime \prime} \times 0.86^{\prime \prime}$ & $15^{\circ}$ & $60 \mu \mathrm{Jy}$ & $2.14^{\prime \prime} \times 1.59^{\prime \prime}$ & $17^{\circ}$ & $60 \mu \mathrm{Jy}$ \\
NGC 7582 & 1994 Nov. 12 & $2326-477$ & $1.36^{\prime \prime} \times 0.92^{\prime \prime}$ & $-2^{\circ}$ & $40 \mu \mathrm{Jy}$ & $2.47^{\prime \prime} \times 1.72^{\prime \prime}$ & $-2^{\circ}$ & $40 \mu \mathrm{Jy}$ \\
Circinus & 1994 Nov. 14 & $1236-684$ & $1.06^{\prime \prime} \times 0.91^{\prime \prime}$ & $-39^{\circ}$ & $45 \mu \mathrm{Jy}$ & $1.92^{\prime \prime} \times 1.64^{\prime \prime}$ & $-36^{\circ}$ & $100 \mu \mathrm{Jy}$ \\
\hline \hline
\end{tabular}


TABLE 2. Measurements

\begin{tabular}{|c|c|c|c|c|c|c|c|c|}
\hline Galaxy & $\underset{(\mathrm{mJy})}{2^{\prime \prime}}$ & Sp. Index & $\underset{\text { (mJy) }}{6^{\prime \prime}}$ & Sp. Index & $\begin{array}{c}\text { Total } \\
6 \mathrm{~cm} \text { flux } \\
\text { (m.Jy) }\end{array}$ & $\begin{array}{l}\text { Single-dish } \\
\text { 6cm flux } \\
\text { (mJy) }\end{array}$ & $q$ & $\begin{array}{c}\mathrm{T}_{b} \\
(\mathrm{~K})\end{array}$ \\
\hline NGC 1365 & 4.6 & -1.1 & 10 & -1.5 & 154 & 230 & 2.28 & 138 \\
\hline NGC 4945 & 275 & -0.3 & 1270 & -0.8 & 2370 & 3055 & 1.88 & 8280 \\
\hline NGC 6221 & 4.0 & -0.5 & 14 & -0.6 & 189 & 164 & 2.21 & 120 \\
\hline NGC 6810 & 2.5 & -0.2 & 5.7 & -0.1 & 73 & 72 & 2.22 & 75 \\
\hline NGC 7582 & 11.2 & -0.7 & 39 & -0.5 & 84 & 143 & 2.32 & 337 \\
\hline Circinus & 21.8 & -0.01 & 49 & -0.2 & 372 & 695 & 2.31 & 656 \\
\hline
\end{tabular}

Notes to Table 2.

Galaxy name, 3 and $6 \mathrm{~cm}$ fluxes in a $2^{\prime \prime}$ and 6 "diameter aperture (typicall error $\pm 0.1 \mathrm{mJy}$ ), 3 and $6 \mathrm{~cm}$ spectral index after smoothing the $3 \mathrm{~cm}$ map to the $6 \mathrm{~cm}$ resolution (error \pm 0.1 ), total $6 \mathrm{~cm}$ flux from our images (error $\pm 1 \mathrm{mJy}$ ), single-dish 6cm flux from Wright et al. (1994, 1996), q parameter measuring the ratio of Far-IR to radio flux and brightness temperature (see text for details). 
Table 3. Derived Quantites

\begin{tabular}{ccccccccccc}
\hline \hline Galaxy & Type Distance Scale & $\begin{array}{c}\text { Incl. } \\
(\mathrm{Mpc})\end{array}$ & $\left(\mathrm{pc} /{ }^{\prime \prime}\right)$ & $\left(^{\circ}\right)$ & $\begin{array}{c}\log \mathrm{L}_{6 \mathrm{~cm}} \\
(\mathrm{erg} / \mathrm{s})\end{array}$ & $\begin{array}{c}\log \mathrm{L}_{F e} \\
(\mathrm{erg} / \mathrm{s})\end{array}$ & $\begin{array}{c}\log \mathrm{L}_{B r \gamma} \\
(\mathrm{erg} / \mathrm{s})\end{array}$ & $\begin{array}{c}\dot{N}_{6 \mathrm{~cm}} \\
(/ \mathrm{yr})\end{array}$ & $\begin{array}{c}\dot{N}_{F e} \\
(/ \mathrm{yr})\end{array}$ \\
\hline \multirow{2}{*}{ NGC 1365 } & $\mathrm{SBb}$ & 20.0 & 97 & 60 & 37.36 & 39.09 & 39.15 & 0.02 & 0.03 \\
NGC 4945 & $\mathrm{SBcd}$ & 6.7 & 32 & 78 & 38.51 & 38.31 & 38.56 & 0.23 & 0.005 \\
NGC 6221 & $\mathrm{SBbc}$ & 24.0 & 116 & 43 & 37.67 & 39.40 & 39.37 & 0.03 & 0.06 \\
NGC 6810 & Sab & 36.0 & 175 & 74 & 37.63 & 39.38 & 38.92 & 0.03 & 0.06 \\
NGC 7582 & SBab & 29.9 & 145 & 64 & 38.30 & 39.65 & 39.57 & 0.14 & 0.11 \\
Circinus & $\mathrm{Sb}$ & 4.0 & 19 & 60 & 36.30 & 38.04 & - & 0.002 & 0.003 \\
& & & & & & & & & \\
\hline \hline
\end{tabular}

Notes to Table 3 .

Galaxy name, type, distance, physical scale, inclination, $6 \mathrm{~cm}$, [FeII] and $\mathrm{Br} \gamma$ luminosity in a 6 " diameter aperture (typical measurement error is $1 \%$ for radio and $5 \%$ for line fluxes), $6 \mathrm{~cm}$ and $[\mathrm{FeII}]$ derived $\mathrm{SN}$ rates (see text for details). 